\title{
BMJ Open Retrospective review of nicotine exposures in California from 2012 to 2018 and analysis of the impacts of e-cigarette regulations
}

\author{
Gabrielle Driller, Emily Plasencia, Dorie E Apollonio (D)
}

To cite: Driller G, Plasencia E, Apollonio DE. Retrospective review of nicotine exposures in California from 2012 to 2018 and analysis of the impacts of e-cigarette regulations. BMJ Open 2021;11:e043133. doi:10.1136/

bmjopen-2020-043133

- Prepublication history and additional material for this paper are available online. To view these files, please visit the journal online (http://dx.doi. org/10.1136/bmjopen-2020043133).

Received 29 July 2020 Revised 04 February 2021 Accepted 15 February 2021

Check for updates

(C) Author(s) (or their employer(s)) 2021. Re-use permitted under CC BY-NC. No commercial re-use. See rights and permissions. Published by BMJ.

Department of Clinical Pharmacy, University of California San Francisco, San Francisco, California, USA

Correspondence to Dr Dorie E Apollonio; dorie.apollonio@ucsf.edu

\section{ABSTRACT}

Objectives To review the association between US ecigarette regulations and the number of reported nicotine exposures, and identify higher-risk products

Design Retrospective review of de-identified medical records.

\section{Setting California}

Participants Cases reported to California Poison Control System in 2012-2018.

Primary and secondary outcome measures Suspected nicotine toxicity; route of exposure and product characteristics.

Results We examined 5277 exposures, of which 3033 involved combustible cigarettes, 1489 involved e-cigarettes and 818 involved other substances (ie, chewing tobacco, nicotine patches, nicotine lozenges, hookah, etc). Implementation of the Child Nicotine Poisoning Prevention Act of 2015 was not significantly associated with reduced exposures. Exposures for ecigarettes increased significantly after the 2017 Food and Drug Administration Compliance Policy $(\mathrm{p}=0.003$, coefficient $($ coeff $)=0.61)$. Total exposures for all tobacco and nicotine products also increased significantly after the policy change $(p=0.01$, coeff $=1.26)$. Nicotine exposure outcomes classified as being of minor and moderate severity increased significantly after implementation of the 2017 Compliance Policy $(p=0.004$, coeff $=0.54$ and $p=0.002$, coeff $=0.56$, respectively). Ingestion was the most common route of exposure (87.7\%), followed by inhalation (8.1\%), dermal (6.5\%), ocular $(2.1 \%)$ and other (intranasal, rectal, sublingual and unknown) routes $(0.2 \%)$; some cases reported multiple routes of exposure. Exposure cases involving e-cigarettes fell into three problem categories: product design, labelling and the appeal of flavours.

Conclusions Our analysis found that despite previous studies suggesting that the Child Nicotine Poisoning Prevention Act appeared to have reduced exposures for e-cigarettes, there was no significant change in exposures after its implementation. In contrast, there was a $30 \%$ increase in California e-cigarette exposures following the 2017 Compliance Policy. We conclude that current regulations are insufficient to reduce nicotine toxicities due to e-cigarette use.
Strengths and limitations of this study

- A major strength of this study is that it reviews exposures before and after the institution of recent US federal regulations on e-cigarettes that have not been previously studied.

- We drew on data from records that reflect real-world exposures to nicotine.

- We are limited by the fact that cases reports are based on patient and provider knowledge of the California Poison Control System (CPCS) and willingness to call, and that some cases were lost to follow-up.

- The cases reported to CPCS are likely an underestimate of the total amount of all nicotine exposures.

\section{INTRODUCTION}

Nicotine poisonings, linked to liquid nicotine used in e-cigarettes, have increased significantly over the past decade in the USA. ${ }^{12}$ The annual number of exposure cases involving e-cigarettes recorded in the National Poison Data System increased $4990 \%$ between 2010 and 2018, from 57 in 2010 to 2901 in $2018 .^{3}$ With this surge in poisonings comes cause for concern, e-cigarette exposures in children under 6 years of age were found to be 5.2 times more likely to result in healthcare facility admissions and 2.6 times more likely to result in severe outcomes than cigarette exposures. ${ }^{4}$

Acute nicotine toxicity manifests in a biphasic pattern. ${ }^{5}$ Initial symptoms include tachycardia, nausea, vomiting, salivation, hypertension, tremors and seizures. ${ }^{5-7}$ Later symptoms include bradycardia, hypotension, respiratory depression, muscle paralysis and coma. ${ }^{5-7}$ The ingestion of even small doses of nicotine can have toxic effects. Conservative estimates of the LD50 for nicotine are around $30-60 \mathrm{mg}$ in adults and $10 \mathrm{mg}$ in children. ${ }^{6-8}$ Some e-cigarette companies (including Juul) sell liquid nicotine cartridges containing 
$3-5 \%$ nicotine. ${ }^{910}$ Manufacturer statements indicate that the amount of nicotine in one $5 \%$ cartridge is about 40 $\mathrm{mg}$ and $23 \mathrm{mg}$ for a $3 \%$ cartridge. $^{9}$ At $40 \mathrm{mg}$ for a $5 \%$ cartridge, a child would need to ingest just one-fourth of the cartridge to reach this suspected oral LD50.

E-cigarettes were largely unregulated prior to 2016 when two interventions were introduced, the Child Nicotine Poisoning Prevention Act (CNPPA) and the Final Deeming Rule. The CNPPA (effective 26 July 2016) requires liquid nicotine containers used in open-tank systems to be specially packaged to prevent children under the age of 5 years from accessing their contents. ${ }^{11}$ This legislation did not apply to sealed, pre-filled and disposable liquid nicotine containers such as Juul and Puff Bar, two popular e-cigarette devices among adolescents. ${ }^{11}{ }^{12}$ While e-cigarette and liquid nicotine exposure calls reported to poison control centres dropped slightly after the implementation of the CNPPA, they increased by $217 \%$ from 2017 to $2019 .{ }^{13}$ This significant increase in exposures could be explained by the lack of childresistant packaging regulations for pod-based disposable liquid nicotine containers, as these were exempt from the CNPPA.

In May 2016, the Final Deeming Rule was issued by the Food and Drug Administration to extend its tobacco product authority to include e-cigarettes. ${ }^{14}{ }^{15}$ In order to remain on the market, this legislation required e-cigarettes and liquid nicotine products to undergo premarket review based on product ingredients, health impacts, design and appeal to youth and non-users. ${ }^{15}$ Originally the deadline for e-cigarette companies to submit their applications for premarket review was 8 August 2018, however in August 2017, this deadline was later extended to 8 August 2022. ${ }^{14}{ }^{16}$ This extension, referred to as the 2017 Compliance Policy, provided e-cigarette companies an additional 4 years to remain on the market, largely unregulated, while preparing for premarket authorisation. E-cigarette sales drastically increased in the second half of 2018, a trend that continued into 2019. ${ }^{17} 18$

Since the implementation of the 2017 Compliance Policy, no studies have been conducted to assess its potential impact on nicotine exposures. Previous studies assessing e-cigarette exposures were largely conducted prior to the popularity of newer generation e-cigarettes, and many did not assess the proportion of cases reported among children relative to adults. ${ }^{13}{ }^{19}$ In this study, we sought to address these gaps in research and provide guidance for developing public health responses and potential regulations using data drawn from poison control calls.

We reviewed the association between e-cigarette regulations and the number of reported nicotine exposures, specifically focusing on the CNPPA and the 2017 Compliance Policy. We hypothesised that e-cigarette exposures increased after the 2017 Compliance Policy (given that the postponement allowed e-cigarette producers to sell their products with limited regulation), and that these effects would exceed any reduction in poisonings achieved after the implementation of the CNPPA. We used exposures from combustible tobacco and other nicotine-containing products as comparison cases, given that these should not have been affected by regulatory changes specific to e-cigarettes. Our study focused on California, where the most popular of these new products, Juul, was first developed and marketed, increasing the likelihood of a rapid response to regulatory changes. Our findings offer insights into the effects of regulatory changes that can guide public health policymaking for new nicotine-containing products and the development of potential safety measures to protect against nicotine toxicities.

\section{METHODS}

We conducted a retrospective review of all suspected nicotine toxicity cases reported to the California Poison Control System (CPCS) from 1 September 2012 to 31 December 2018. The CPCS is a 24-hour helpline that serves as California's main source of phone consultations and recommendations regarding poisonings for the public and health professionals.

Our data consists of de-identified case records that were extracted from the CPCS database using American Association of Poison Control Centers (AAPCC) generic codes such as: chewing tobacco, cigarettes, cigars, e-cigarettes, unknown types of tobacco products and other types of tobacco products. The complete list is given in the online supplemental file. Case records were written and coded by CPCS staff. Due to variability in case coding across CPCS staff, detailed phone records were reviewed by two of the authors (EP and GD) and recoded for characteristics and outcomes. Study data was collected and managed using REDCap (Research Electronic Data Capture), a secure, web-based application designed to support data capture. ${ }^{20} 21$ Disagreements were resolved by consulting with a third author (DA).

Cases were excluded if they met one or more of the following exclusion criteria: exposure location outside of California, duplicate case, non-human exposure, confirmed non-exposure and unrelated effect (see online supplemental file for examples). 'Unrelated effect' was applied to cases where the effect was deemed unlikely to be caused by the nicotine exposure. This included cases with long durations (eg, weeks, months) between the nicotine exposure and reported effect, and those in which there were other, non-nicotine reported substances that were more likely to have contributed to the outcome. All cases were reviewed and recoded by date of exposure, age, sex, city, type of nicotine product(s), exposure to other non-nicotine products, route of exposure, symptoms, outcome and hospitalisation (if applicable).

The primary outcome measured was nicotine toxicity reported to the CPCS. This outcome was stratified by potential associations consisting of e-cigarette use (all e-cigarette devices and liquid nicotine products), combustible tobacco use (cigarettes, cigars and loose tobacco) 
and other nicotine product use (chewing tobacco, nicotine patches, nicotine gum/lozenges and hookah products). Outcomes were also stratified by time: before and after implementation of the CNPPA, and before and after the 2017 Compliance Policy. Secondary outcomes included the degree of severity, route of nicotine exposure and age of the patient.

Outcomes were placed into the following categories: non-toxic/no effect, minor effect, moderate effect, major effect, death, unable to follow but potentially toxic exposure, unable to follow but likely not toxic exposure and other. Non-toxic/no effect was used to code all cases that stated the patient was asymptomatic. Minor effect included cases in which the patient only experienced symptoms from one category (gastrointestinal, nervous system, cardiovascular, pulmonary, dermal, renal, ocular or miscellaneous) and the CPCS did not refer to a healthcare provider. Moderate effect included cases in which symptoms from more than one category were observed, or the CPCS recommended an immediate visit to the emergency department or an MD. Major effect included cases in which any one of the following symptoms were observed: loss of consciousness, seizure, haematemesis or haemoptysis, as well as cases that resulted in hospital admission (except for the psychiatric ward). Cases that did not mention any symptoms and did not explicitly state that the patient was asymptomatic were coded as unable to follow. These cases were subdivided into potentially toxic or likely not toxic, depending on the amount of nicotine product ingested and the CPCS staff evaluation of potential toxicity when included in the note (for children less than 5 years old, ingestion less than one cigarette or three cigarette butts is considered likely subtoxic). Further details on the coding criteria for each category are given in the online supplemental file.

To assess whether observed changes in average monthly exposures were statistically significant, we conducted interrupted time series analyses (ITSA) assessing three periods: before the CNPPA, after its implementation and after the 2017 Compliance Policy. The 'standard ITSA regression model' for a single group follows the form $Y_{t}=$ $\beta_{0}+\beta_{1} T_{t}+\beta_{2} X_{t}+\beta_{3} X_{t} T_{t}+\epsilon_{t}$, where $\beta_{0}$ is the intercept, $\beta_{1}$ is the slope prior to intervention, $\beta_{2}$ is the change immediately following the intervention and $\beta_{3}$ represents the treatment effect of that intervention over time. ${ }^{22}$ Multiple group analyses expand the regression model to include four additional terms $\left(\beta_{4}-\beta_{7}\right)$ that represent differences between groups. ${ }^{22}$ We used the 'itsa' plugin for Stata to conduct the analysis, followed by 'actest' to test model assumptions, as well as additional sensitivity analyses based on censored dates. ${ }^{22}$ Our subgroup analysis compared trends in exposures for e-cigarettes to trends in exposures for combustible tobacco products and other nicotinecontaining products by conducting separate interrupted time series for each product type.

\section{Patient and public involvement}

No patient involved.
RESULTS

From 1 September 2012 to 31 December 2018, there were 6193 nicotine exposures reported to the CPCS, of which 916 were excluded based on study criteria. Of the 5277 included exposure cases, 3033 involved combustible cigarettes, 1489 involved e-cigarettes and 818 involved other substances (ie, chewing tobacco, nicotine patches, nicotine lozenges, hookah, etc). Some cases included multiple substances, either from various nicotine products or nonnicotine products (ie, prescription medication, illicit drugs, cleaning products, etc). Of the cases with documented symptoms, $49.4 \%$ had minor outcomes, $47.9 \%$ had moderate outcomes and $2.8 \%$ had major outcomes. Nicotine-related exposures reported to CPCS during this time period were primarily for children; $76.1 \%$ of all exposures were in children under 5 years of age, and $4.5 \%$ were in children between 5 and 18 years of age. Children under 5 years of age accounted for $70.8 \%$ of all e-cigarette exposures. While CPCS data typically does not refer to products by brand, 10 cases specifically mentioned 'Juul', all in 2017 or 2018.

We report the distribution of exposures over time and around the periods of regulatory changes. Following the implementation of the CNPPA, the average monthly exposures from e-cigarettes increased from 18.2 to 21.2, as shown in table 1. After the 2017 Compliance Policy went into effect, the average monthly exposures for e-cigarettes increased by $30 \%$, from 18.4 to 24.0. In contrast, the average monthly exposures for combustible cigarettes decreased from 41.5 to 34.2 .

Our interrupted time series analysis of these exposures found that, despite previous reports suggesting that the CNPPA appeared to have reduced exposures for e-cigarettes, this change was not statistically significant, as shown in figure 1 . There was also no significant change in exposures for combustible tobacco products, other nicotine-containing products or for all tobacco and nicotine-containing products after implementation of the CNPPA.

In contrast, exposures for e-cigarettes increased significantly after the 2017 Compliance Policy $(p=0.003$, coefficient $($ coeff $)=0.61)$. Exposures for both combustible tobacco products and other nicotine-containing products did not change significantly after this regulatory shift, as anticipated given that the policy changes specifically addressed e-cigarettes. The shift in e-cigarette exposures was substantial enough in scope that total exposures containing all types of tobacco and nicotine products increased significantly after the policy change $(\mathrm{p}=0.01$, coeff $=1.26$ ). Visual inspection of exposures in figure 1 suggested that they may have been increasing prior to July 2014, followed by declines after that date, an observation that raised concern about possible model misspecification. Our follow-up sensitivity analyses considered more limited time periods by censoring the data from July 2014 forward and found comparable results with no changes in significance (although with larger CIs due to 
Table 1 Number of nicotine exposures reported to the California Poison Control System, 2012-2018

\section{Before Child Nicotine Poisoning \\ Prevention Act of 2015}

Dates: 1 September 2012-27 January 2016
After Child Nicotine Poisoning Prevention Act of 2015

Dates: 28 January 2016-31 December 2018

\begin{tabular}{llllll} 
& Total exposures & $\begin{array}{l}\text { Average number of } \\
\text { monthly exposures }\end{array}$ & Total exposures & $\begin{array}{l}\text { Average number of } \\
\text { monthly exposures }\end{array}$ \\
\hline Combustible tobacco products & 1672 & 40.8 & 1364 & 39.0 \\
\hline E-cigarettes & 745 & 18.2 & 10.6 & 341 & 21.2 \\
\hline Other nicotine-containing products & 433 & 377 & 10.8
\end{tabular}

\begin{tabular}{|c|c|c|c|c|}
\hline & \multicolumn{2}{|c|}{ Before August 2017 Compliance Policy } & \multicolumn{2}{|c|}{ After August 2017 Compliance Policy } \\
\hline & $\begin{array}{l}\text { Dates } \\
2017\end{array}$ & 2012-9 August & $\begin{array}{l}\text { Dates } \\
2018\end{array}$ & 7-31 December \\
\hline Combustible tobacco products & 2489 & 41.5 & 547 & 34.2 \\
\hline E-cigarettes* & 1103 & 18.4 & 383 & 24.0 \\
\hline Other nicotine-containing products & 639 & 10.7 & 171 & 10.7 \\
\hline
\end{tabular}

The average number of monthly exposures was not adjusted for increasing population size. Information regarding the calculation of these values is given in the online supplemental file.

*Includes liquid nicotine exposures.

reduced sample size); as a result, figure 1 includes the full time series.

When analysing secondary outcomes for age, there were no significant changes in exposures for any nicotine substance in children under the age of 5 years as compared with those who were 5 years or older. When
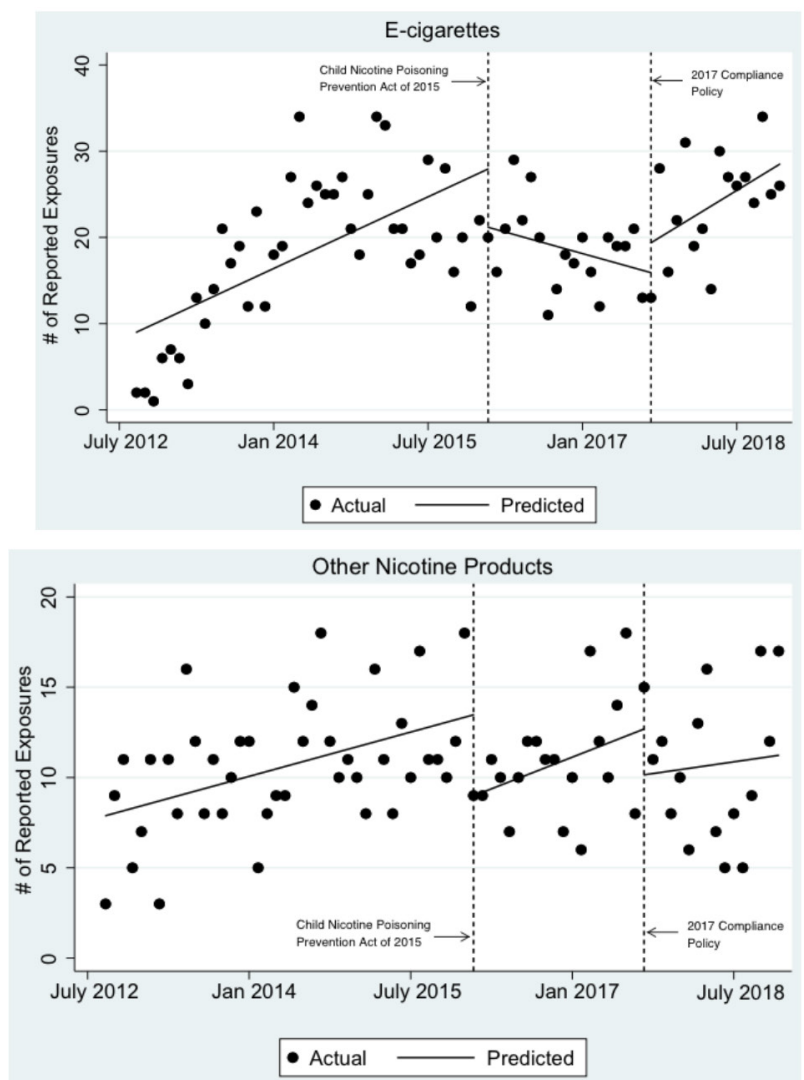

analysing for outcome (minor, moderate and major), minor and moderate outcomes reported for all nicotine exposures increased significantly after implementation of the 2017 Compliance Policy $(\mathrm{p}=0.004$, coeff $=0.54$ and $\mathrm{p}=0.002$, coeff $=0.56$, respectively). Likewise, minor outcomes reported for e-cigarette exposures increased
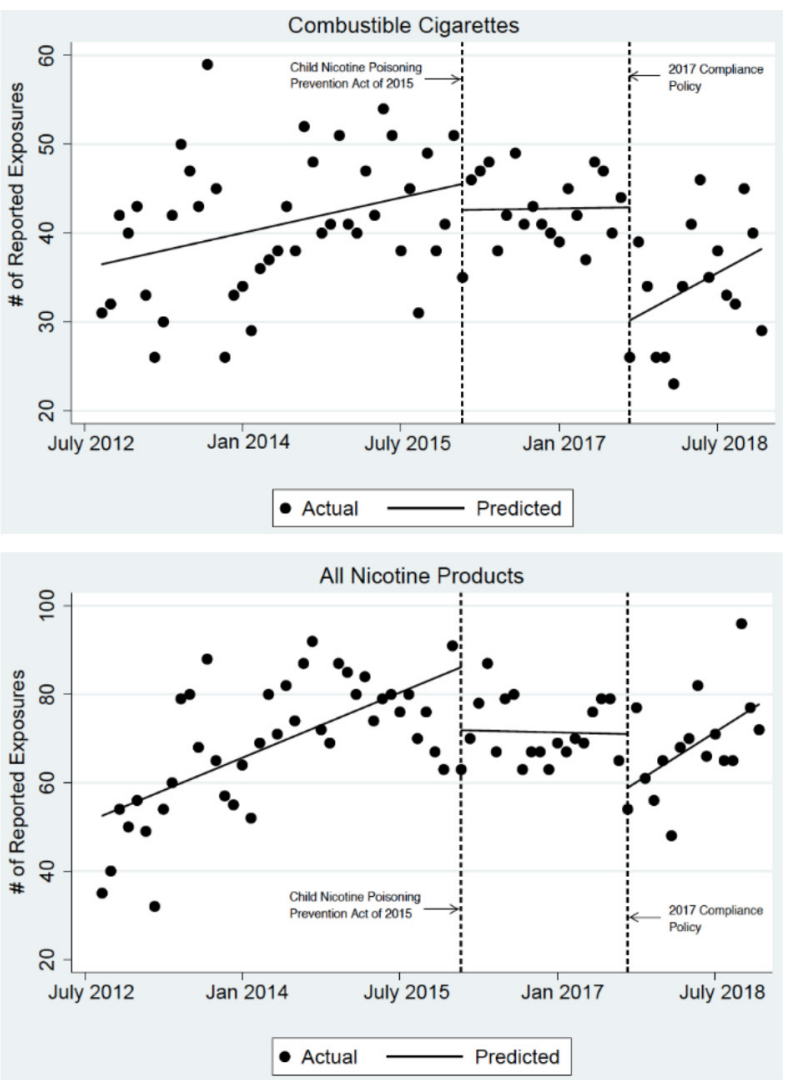

Figure 1 Nicotine exposures by substance. Source: California Poison Control System. 


\section{All Nicotine Product Exposures}

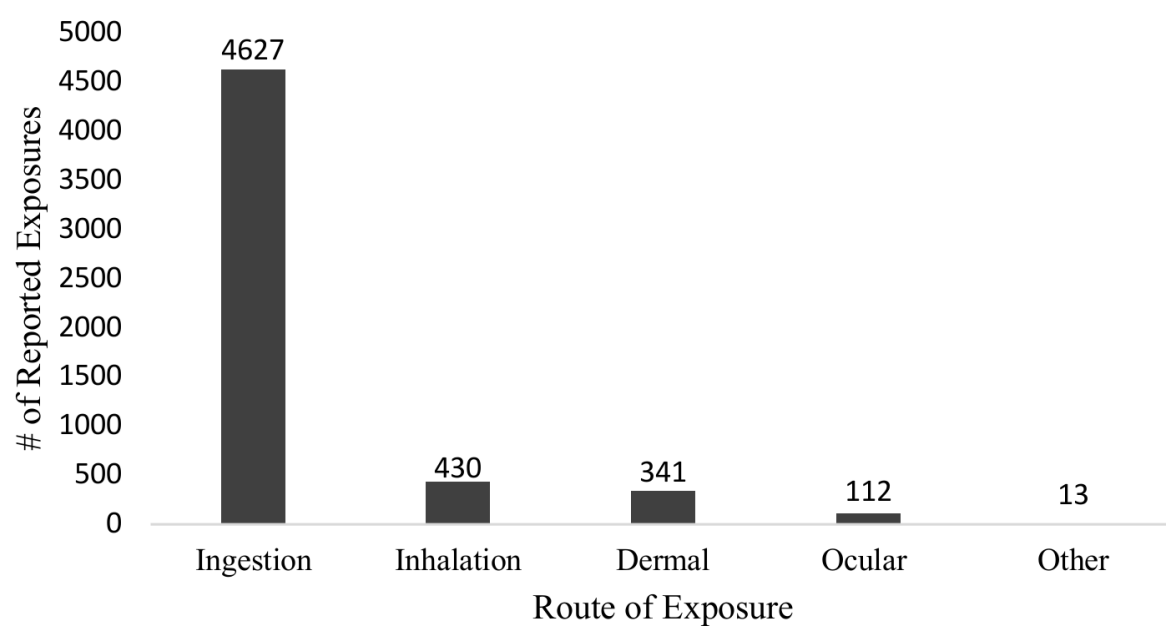

Figure 2 Nicotine exposures reported to California Poison Control System (CPCS) by route, 2012-2018. Source: CPCS data coded by the authors.

significantly after implementation of the policy $(\mathrm{p}<0.001$, coeff $=0.39$ ), however, there was no significant change in e-cigarette exposures with moderate outcomes. Detailed data on nicotine exposures by substance and subdivided by minor and moderate outcomes are given in the online supplemental file. Comparisons of trends in major outcome cases could not be performed due to the low number of exposures.

There has been limited reporting on the characteristics of exposures for e-cigarettes and other nicotinecontaining products; as a result, we offer details on the nature of these exposures. Overall, ingestion was the most common route of exposure $(87.7 \%)$, with inhalation $(8.1 \%)$, dermal $(6.5 \%)$, ocular $(2.1 \%)$ and other routes following accordingly, as shown in figure 2. Some cases consisted of multiple routes of exposure. 'Other' routes of ingestion consisted of intranasal, rectal, sublingual and unknown (when the case lacked information pertaining to the route of exposure). In all cases in which it was observed or assumed that the patient put a nicotine product in their mouth, regardless of if it was swallowed, the route was considered to be ingestion.

Exposure cases involving e-cigarettes can be grouped into three problem categories: product design, labelling and the appeal of flavours. For young children (under 5 years of age), situations relating to product design included those in which children were able to access e-cigarette cartridges or refill containers, resulting in ingestion, inhalation, ocular and dermal exposures. In many of these cases, the product leaked or residue was found on the outer surface of the e-cigarette or refill bottle, leading to both dermal and ingestion exposures when the child grabbed the item and licked or placed it in their mouth. The appeal of flavours category included cases in which children mistook liquid nicotine for candy. In one example, nine children in the sixth grade went directly from school to the emergency department after ingesting liquid nicotine flavoured as 'Bazooka Sour Straws'.
In adults, liquid nicotine exposures mainly related to complications with product design. Cases included situations in which liquid nicotine leaked out of cartridge reservoirs resulting in dermal exposures and ingestion when the user inhaled. Additionally, splashes of liquid nicotine created in the process of opening e-cigarette cartridge reservoirs resulted in dermal, ocular and ingestion exposures. Explosions of e-cigarettes were also documented, resulting in dermal, ocular and ingestion exposures. Lastly, broken e-cigarettes and liquid nicotine containers stored within users' clothing pockets resulted in dermal exposures.

In individuals of all ages, labelling issues resulted in accidental ingestion, dermal and ocular exposures when liquid nicotine was mistaken for other liquids (eg, gripe water, CBD liquid, herbal supplements, antibiotic eye drops and re-wetting eye drops). Figure 3 depicts the similarities between liquid nicotine bottles and non-nicotine containing products. In some cases, labelling issues (either due to the nature of the label or its deterioration over time) meant that patients, caregivers and healthcare providers were unable to quantify the amount of nicotine involved in the exposure. Cases where the total volume and/or nicotine concentration was absent on labels for liquid nicotine were reported to CPCS by both patients and healthcare providers.

\section{DISCUSSION}

Our findings revealed a $30 \%$ increase in California e-cigarette exposures following the 2017 Compliance Policy. While our analysis did not control for population growth, the increase in exposures is much larger than the $4 \%$ increase in California's population between 2012 and 2018. Such a significant increase in e-cigarette poisonings is particularly concerning given that the population at risk of exposure is vulnerable to regulatory delay; over three-quarters of all 


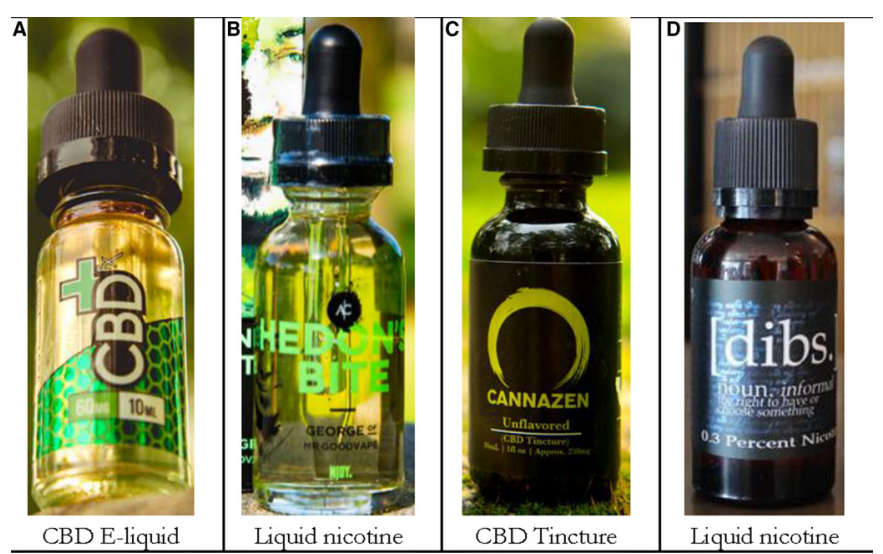

Figure 3 E-liquid containers compared with non-nicotine containing products. Sources: (A) 'CBD E-Liquid, Tinctures' by Vaping360 is licensed with CC BY 2.0. To view a copy of this license (visit https://creativecommons.org/licenses/ by/2.0/). (B) 'E-liquid bottle Njoy' by Sarah-Johnson is licensed with CC BY 2.0. To view a copy of this license (visit https://creativecommons.org/licenses/by/2.0/). (C) 'CBD ELiquid, Tinctures' by Vaping360 is licensed with CC BY 2.0. To view a copy of this license (visit https://creativecommons. org/licenses/by/2.0/). (D) 'Vaporizer, E-Liquids' by Lindsay Fox is licensed with CC BY 2.0. To view a copy of this license (visit https://creativecommons.org/licenses/by/2.0/).

exposures occurred among children under the age of 5 years. This proportion is even larger than that seen in previous nationwide poison control centre studies. One study conducted in 2019 found that less than twothirds $(65 \%)$ of e-cigarette exposure cases were in children under the age of 5 years; while our study found that $71 \%$ of e-cigarette exposure cases were in children of the same age group. ${ }^{3}$ The higher rate of exposures among children under the age of 5 years seen in our data may be explained by the extent of products included and potentially by study location. Our California focus may have led us to observe the impacts of e-cigarettes on an earlier timeline, since Juul was founded and first marketed in California, colloquially referred to as 'ground zero of the vaping epidemic'. ${ }^{23}$

Our review of the characteristics of e-cigarette exposures suggests that they fall into three main problem categories: product design, labelling and flavours. These observations suggest strategies for future regulation that could more effectively address these increased exposures. The CNPPA focused on product design affecting only open-tank systems, for which users refill a reservoir using a container of liquid nicotine. ${ }^{11}$ The shift to newer-generation e-cigarettes that use prefilled pods or are produced as single-use devices means that these design regulations are irrelevant to products popular in 2017 and beyond. Problems with product design that resulted in exposures with newer devices consisted of issues of fragility (eg, broken reservoirs) and accessibility to young children (eg, cartridges that were either not properly stored or disposed). Specific syringes feature retractable needle mechanisms to protect against needlestick injury after use; similar product design modifications could be used to prevent access to e-cigarette cartridge reservoirs after disposal. Additionally, strategies used for disposal of hazardous substances and objects, such as sharps containers, could be implemented for nicotine-containing products to reduce access by young children.

The labelling of liquid nicotine products is of particular concern given that users have mistaken nicotine solutions for other medications and products. Regulations pertaining to the durability of labels as well as more thorough enforcement of current regulations would help to promote e-cigarette product safety. Additionally, the deterioration of nicotine-containing product labels can result in situations in which the containers lack information about the amount of nicotine contained. Further regulations could help to ensure label stability in order to ensure that patients, caregivers and healthcare providers are able to quantify the amount of nicotine ingested in cases of possible toxicity, thereby allowing more rapid and accurate treatment.

Although flavours other than menthol and tobacco were regulated for cartridge-based e-cigarette products in early 2020, flavours were still allowed for single-use and open-tank systems. ${ }^{15}$ Companies continue to sell fruit and candy flavours that appeal to younger teens and children. Allowing the sale of flavoured products is likely to result in persistent poisonings in young children mistaking nicotine solution for candy and increases the likelihood that these children will consume more nicotine, resulting in higher risk of serious health outcomes. A flavour ban for all nicotine products could help minimise the quantity and severity of nicotine exposures in young children and teens. Increasing public awareness to the risk of nicotine toxicity from these flavoured e-cigarettes by increasing mass-communication campaigns and creating schoolbased prevention policies and interventions could further minimise exposures in teens.

Our study has limitations. Some outcomes could not be tracked due to incomplete clinical data documentation and follow-up. Exposure reports made to poison control centres, including CPCS, are reliant on a patient's knowledge of these centres and willingness to call. As a result, the cases reported are likely an underestimate of the total amount of all nicotine exposures. Limited reporting, however, would make it more difficult to observe a relationship between regulatory changes and associated outcomes, suggesting that the relationships we identified may be underestimates of health risks. An additional limitation is reliance on information provided by callers, which may lead to inaccurate estimates of the level of exposure in unobserved cases, or underestimates of exposure in cases where callers are concerned about the potential ramifications of the call, particularly given that most exposures were in young children. In cases without follow-up by a healthcare provider, exposure data were primarily based on self-report rather than urine or blood 
test confirmation. Our study design was observational and did not control for population increases or changes in prevalence (California surveillance data report estimated prevalence of use on an annual basis). However some existing research considers sales of e-cigarettes, although these data exclude multiple types of retailers (vape shops, Internet), and has found that sales remained relatively stable for several months after the policy change, ${ }^{18}$ even though exposures spiked immediately. Given limitations of study design we identify associations but cannot prove causality; nonetheless, the significant increase in exposures beginning in 2017 is concerning regardless of its underlying cause.

Our findings related to major outcomes were limited based on our sample size. Future work could analyse nationwide data to assess whether outcomes such as hospital admissions increased significantly following the 2017 Compliance Policy. Additionally, studies should continue to assess the impact of new regulations relating to nicotine products, including e-cigarettes, on the rate of suspected nicotine poisonings.

\section{CONCLUSION}

Our findings suggest that existing efforts to regulate e-cigarettes have failed to prevent poisonings and that young children are particularly at risk. Passage of the CNPPA was not associated with a significant decline in exposures, while e-cigarette exposures significantly increased following the 2017 Compliance Policy. Our review of the characteristics of exposures suggests that the CNPPA was ineffective due to its limited scope, which addressed only liquid nicotine refill containers for use in open-tank systems, and that the guidance corresponding to liquid nicotine flavourings is likely to be ineffective given that it applies only to cartridgebased e-cigarette products. Our review of exposure characteristics highlights potential strategies that could mitigate exposures using existing regulations for other products. These include regulations pertaining to product design, product disposal and labelling. Given the substantial increase in nicotine exposures in a highly vulnerable population, implementing these changes has the potential to protect the public against the harmful impacts of liquid nicotine and address the rapid rise in poisonings associated with vaping.

\section{Twitter Dorie E Apollonio @apollonio}

Acknowledgements The authors acknowledge Dr Raymond Ho for his assistance in gathering California Poison Control System data for nicotine exposures and his insight on data collection and analysis.

Contributors GD, EP and DEA all worked together to design the study, interpret the results and revise the manuscript. GD and EP drafted the manuscript and DEA analysed the data.

Funding This work was supported by California Tobacco-Related Disease Research Program \#25IR-0025 and \#26IR-0014 (Apollonio). The funders had no role in the design or conduct of the study.

Competing interests None declared.
Patient consent for publication Not required.

Ethics approval This study was approved by the Institutional Review Board at the University of California, San Francisco, on 29 January 2020 (\#19-29544).

Provenance and peer review Not commissioned; externally peer reviewed.

Data availability statement Data may be obtained from a third party and are not publicly available. Data can be obtained by submitting a Data Request Form to the California Poison Control System.

Supplemental material This content has been supplied by the author(s). It has not been vetted by BMJ Publishing Group Limited (BMJ) and may not have been peer-reviewed. Any opinions or recommendations discussed are solely those of the author(s) and are not endorsed by BMJ. BMJ disclaims all liability and responsibility arising from any reliance placed on the content. Where the content includes any translated material, BMJ does not warrant the accuracy and reliability of the translations (including but not limited to local regulations, clinical guidelines, terminology, drug names and drug dosages), and is not responsible for any error and/or omissions arising from translation and adaptation or otherwise.

Open access This is an open access article distributed in accordance with the Creative Commons Attribution Non Commercial (CC BY-NC 4.0) license, which permits others to distribute, remix, adapt, build upon this work non-commercially, and license their derivative works on different terms, provided the original work is properly cited, appropriate credit is given, any changes made indicated, and the use is non-commercial. See: http://creativecommons.org/licenses/by-nc/4.0/.

ORCID ID

Dorie E Apollonio http://orcid.org/0000-0003-4694-0826

\section{REFERENCES}

1 American Association of Poison Control Centers (AAPCC). ECigarettes and liquid nicotine, 2020. Available: https://aapcc.org/ track/ecigarettes-liquid-nicotine [Accessed 2 Feb 2021].

2 Miller A. Nicotine poisoning increase due to e-cigarettes. CMAJ 2014;186:E367.

3 Wang B, Liu S, Persoskie A. Poisoning exposure cases involving ecigarettes and e-liquid in the United States, 2010-2018. Clin Toxicol 2020;58:488-94.

4 Kamboj A, Spiller HA, Casavant MJ, et al. Pediatric exposure to e-cigarettes, nicotine, and tobacco products in the United States. Pediatrics 2016;137. doi:10.1542/peds.2016-0041. [Epub ahead of print: 09 May 2016].

5 Paik JH, Kang S, Durey A, et al. Symptomatic bradycardia due to nicotine intoxication. Rev Bras Ter Intensiva 2018;30:121-6.

6 Mishra A, Chaturvedi P, Datta S, et al. Harmful effects of nicotine. Indian J Med Paediatr Oncol. In Press 2015;36:24-31.

7 Metz CN, Gregersen PK, Malhotra AK. Metabolism and biochemical effects of nicotine for primary care providers. Med Clin North Am 2004;88:1399-413.

8 Centers for Disease Control and Prevention. Nicotine: immediately dangerous to life or health concentrations (idlh), 2014. Available: https://www.cdc.gov/niosh/idlh/54115.html [Accessed 2 Feb 2021].

9 JUUL Labs Inc. Discover more about juulpods \& flavors, 2019. Available: https://www.juul.com/resources/What-is-JUUL-VapeLiquid-All-JUUL-Pod-Flavors?\&ag=CA [Accessed 2 Feb 2021].

10 Centers for Disease Control and Prevention. Sales of juul e-cigarettes skyrocket, posing danger to youth, 2018. Available: https://www.cdc. $\mathrm{gov} / \mathrm{media} / \mathrm{releases} / 2018 / \mathrm{p} 1002-\mathrm{e}-$ Cigarettes-sales-danger-youth. html [Accessed 2 Feb 2021].

11 S.142 - child nicotine poisoning prevention act of 2015. 114th Congress (2015-2016) ed 2016.

12 Kaplan S. Lawmakers say puff bar used pandemic to market to teens. The New York Times, 2020.

13 Govindarajan P, Spiller HA, Casavant MJ, et al. E-Cigarette and liquid nicotine exposures among young children. Pediatrics 2018;141:e20173361.

14 US Food and Drug Administration. Enforcement priorities for electronic nicotine delivery system (ends) and other deemed products on the market without premarket authorization: guidance for industry guidance document, 2020. Available: https://www. fda.gov/regulatory-information/search-fda-guidance-documents/ enforcement-priorities-electronic-nicotine-delivery-system-endsand-other-deemed-products-market [Accessed 2 Feb 2021].

15 US Food and Drug Administration. Fda takes significant steps to protect Americans from dangers of tobacco through new regulation, 2016. Available: https://www.fda.gov/news-events/ 
press-announcements/fda-takes-significant-steps-protectamericans-dangers-tobacco-through-new-regulation [Accessed 2 Feb 2021].

16 US Food and Drug Administration. Extension of certain tobacco product compliance deadlines related to the final deeming rule; withdrawal of guidance, 2020. Available: https://www.federalregister. gov/documents/2020/04/30/2020-09163/extension-of-certaintobacco-product-compliance-deadlines-related-to-the-finaldeeming-rule [Accessed 2 Feb 2021].

17 Liber A, Cahn Z, Larsen A, et al. Flavored e-cigarette sales in the United States under self-regulation from January 2015 through October 2019. Am J Public Health 2020;110:785-7.

18 Ali FRM, Diaz MC, Vallone D, et al. E-cigarette Unit Sales, by Product and Flavor Type - United States, 2014-2020. MMWR Morb Mortal Wkly Rep 2020;69:1313-8.
19 Ordonez JE, Kleinschmidt KC, Forrester MB. Electronic cigarette exposures reported to Texas poison centers. Nicotine Tob Res 2015;17:209-11.

20 Harris PA, Taylor R, Minor BL, et al. The REDCap Consortium: building an international community of software platform partners. $J$ Biomed Inform 2019;95:103208.

21 Harris PA, Taylor R, Thielke R, et al. Research electronic data capture (REDCap)--a metadata-driven methodology and workflow process for providing translational research informatics support. J Biomed Inform 2009:42:377-81.

22 Linden A. Conducting interrupted time-series analysis for single- and multiple-group comparisons. Stata J 2015;15:480-500.

23 Maa J. California is ground zero of the vaping epidemic, 2019. Available: https://www.sfchronicle.com/opinion/openforum/article/ California-is-ground-zero-of-the-vaping-epidemic-14903076.php [Accessed 2 Feb 2021]. 